sample of 13-35-year-old-females and males ( $n \sim 5000)$; (ii) rapid ethnographic community mapping (one urban, one semi urban and two deep rural); (iii) provider and user interviews $(\mathrm{n}=22$ and $\mathrm{n}=58$ respectively); and (iv) group discussion $(n=14)$. All qualitative interviews were audio-recorded, transcribed and analysed using a thematic content analysis. The longitundinal cohorts were used to describe the population awareness and uptake of each of the components of DREAMS at the individual, family and community level, as well as biomedical HIV prevention interventions.

Results 28 services, organised into 10 packages are delivered through 12 implementing partners and three government directorates. In this symposium I describe the population level awareness and uptake of individual, family and community interventions alone and in combination. I also describe the cascade of prevention for specific biomedical interventions, specifically condom use, uptake of HIV testing, voluntary male medical circumcision, HIV Pre-exposure Prophylaxis and treatment, and contraception.

Discussion The HIV prevention cascade is a powerful tool to improve the effective implementation of combination HIV prevention.

Disclosure No significant relationships.

\section{S09.3 APPLICATION OF A CASCADE APPROACH FOR GUIDING PREVENTION OF CONGENITAL SYPHILIS}

Patricia Garcia*. Cayetano Heredia University, Unit of Epidemiology, STIs and HIV, Lima, Peru

10.1136/sextrans-2019-sti.47

Syphilis remains a major public health problem worldwide especially in developing countries and in the region of Latin America and the Caribbean (LAC). Congenital syphilis is caused by mother-to-child transmission of the Treponema pallidum infection during pregnancy. Transmission can occur during any trimester of pregnancy and during any stage of syphilis. Nevertheless, the risk of transmission is highest during early syphilis (primary, secondary, or early latent syphilis). Untreated syphilis in pregnancy can result in adverse pregnancy outcomes including miscarriages, stillbirth, neonatal death, as well as prematurity, low birth weight and other congenital abnormalities. Congenital syphilis is preventable. Treatment to the infected mother with a long acting injectable penicillin (benzathine penicillin $G$ ) can prevent stillbirths and fetal infection if initiated as early as possible during pregnancy or at least 30 days before delivery. Many the countries of the LAC region have committed to reduce cases of congenital syphilis to less than 0.5 cases per 1,000 live births, however many of them are still far from achieving the objective. The reduction or elimination of congenital syphilis can be performed with simple, cost-effective interventions, like screening and treating pregnant women early in antenatal care. Peru has also a commitment to eliminate congenital syphilis and introduced the use of rapid syphilis tests for screening to simplify the testing and improve coverage. However, if a woman is tested and don't receive treatment there is a failure of the system, a broken care continuum. The concept of care cascades has been used to analyze the HIV care continuum and to evaluate other STD control activities. We decided to use Peruvian National data to create a congenital syphilis prevention cascade to visualize the current status of activities and identify opportunities for improvement.

Disclosure No significant relationships.

\section{S09.4 CASCADES AND OTHER APPROACHES TO GUIDE PREP PROGRAMMING}

Sinead Delany-Moretlwe*. Wits RHI, University of the Witwatersrand, Johannesburg, South Africa

\subsection{6/sextrans-2019-sti.48}

Since the 2015 WHO recommendation to offer pre-exposure prophylaxis (PrEP) to all at substantial risk for HIV infection as part of a comprehensive HIV prevention package, there have been significant efforts to expand PrEP access. By 2018, an estimated 465,000 individuals globally had initiated PrEP, a figure that while impressive falls short of the UNAIDS Prevention 2020 target of 3 million eligible people on PrEP by 2020. Achievement of the target will require an intensification of efforts to ensure more effective delivery of PrEP. Similar to the HIV treatment cascade which has been powerful in illustrating the steps needed to achieve viral suppression, several authors have proposed a 'PrEP cascade' which summarises the steps to successful PrEP initiation and in some, continuation. In this presentation we review how these cascades are helpful when populated with programme data for summarising progress as well as identifying points of attrition along the continuum. Using these cascades individual and structural barriers to achieving a particular step can be identified and programmes adapted or strengthened in response. PrEP cascade data can also be used to model the potential impact of PrEP programmes on the HIV epidemic. PrEP cascades also have several unique challenges not observed in treatment cascades. These include the problem of estimating the potential population eligible for PrEP (i.e. denominator), the absence of standard definitions which makes operationalisation within programmes and comparisons across programmes difficult, and the dynamic nature of risk which makes measurement of PrEP continuation a challenge.

\section{S10 - STIS IN LOW AND MIDDLE-INCOME COUNTRIES: RESURGENT INFECTIONS AND EVOLVING CONTROL MEASURES}

\section{Tuesday, July 16, 2019 10:45 AM - 12:15 PM}

\section{S10.1 HIGH RATES OF CURABLE STIS IN LOW AND MIDDLE INCOME COUNTRIES}

Connie Celum*. University of Washington, Global Health, Seattle, USA

\subsection{6/sextrans-2019-sti.49}

Background Sexually-transmitted infections (STIs) increase the risk of infertility and HIV acquisition. Data on STIs in low and middle income countries (LMIC) are sparse because of syndromic management and lack of testing. PrEP projects 
which test for STIs provide key epidemiologic data, identify gaps, and should guide policies.

Methods PrEP projects are reaching populations at risk of HIV in LMIC, including adolescent girls and young women (AGYW), heterosexual HIV serodiscordant couples, and men who have sex with men. STI data from different populations and geographies will be reviewed, and research and intervention gaps will be discussed.

Results Among African AGYW in PrEP projects, the prevalence of Chlamydia trachomatis (CT) and/or Neisseria gonorrhoea (GC) is $30 \%$, most of which is asymptomatic, syphilis is $<1 \%$, and annual incidence of CT is $30 \%$ and GC is $10 \%$, most of which are new infections. Among African heterosexual HIV serodiscordant couples in PrEP studies, the prevalence of CT is $10 \%$, trichomonas is $7 \%$, GC is $6 \%$, and syphilis is $<1 \%$. STI prevalence among African men who have sex with men (MSM) are more sparse, but indicate high prevalence of GC, CT, and syphilis.

Conclusion The prevalence of curable bacterial STIs is high in PrEP projects among diverse populations in LMIC, most of which are missed by syndromic STI management. STI services are an important reproductive sexual health intervention, are valued by clients, and increase the public health impact of PrEP. Interventions to lower the costs of STI diagnostic assays, point of care tests, and treatment of partners are needed. Innovative STI prevention strategies should be evaluated, including doxycycline post-exposure prophylaxis and vaccines. STI testing, treatment, and partner services should be integrated into PrEP, medical male circumcision, antenatal care, and HIV care and prevention programs which reach sexuallyactive persons in LMIC.

Disclosure No significant relationships.

\section{S10.2 BUILDING STD CLINICAL INFRASTRUCTURE IN LMIC - ROLE OF GENERAL VS. SPECIALIZED INFRASTRUCTURE}

Ligang Yang*. Dermatology Hospital, Southern Medical University, STD Dept, Guangzhou, China

\subsection{6/sextrans-2019-sti.50}

Making clinical service for STD patients accessible in LMICs will require to recognize common medical conditions. In China, general hospitals are the most visited by patients, including those with STD. Data from 105 Chinese STD sentinel sites in 2018 showed that $77.2 \%$ of syphilis cases were reported from general hospitals, followed by $5.6 \%$ from women and children hospitals, only $4.7 \%$ of syphilis cases from STD specialized institutions. Community health centers, although providing comprehensive medical services, are generally considered to be of lower level and only 3.0\% syphilis cases were rereported from the community health centers and other primary care centers. In LMICs with limited human resources, general hospitals play very important role in providing clinical services for STD patients but are often unsatisfactory in preventive services. A survey from China found, the rates of contact tracing and partner notification were very low in general hospital as the physicians lack time and willingness for preventive services. The main function for general hospitals, women and child health care hospitals, are to provide STD screening and corresponding treatment for out-patients, inpatients and pregnant women. As for the specialized STD institutions, it usually includes the following functions:1.
Develop appropriate local STD case management procedures (flow chart); 2. STD surveillance, including case auditing and active surveillance; 3 . STD testing center including external STD lab quality assessment; 4. Young people clinics, MSM clinics, et al; 5. Training. To make these services effective, integration of general and specialized institutions at the managerial and administrative levels will be crucial.

Disclosure No significant relationships.

\section{S10.3 CERVICAL CANCER PREVENTION IN LMICS: ARE WE ON THE PATH TO ELIMINATION?}

Megan Huchko*. Duke University, Durham, USA

10.1136/sextrans-2019-sti.51

In May 2018, the World Health Organization announced that a cosmmitment to cervical cancer elimination. In October 2018, modelers from Australia showed that with current rates of vaccine implementation and screening, cervical cancer could be eliminated in that country within the next ten years. Researchers are working on clinical, implementation and modeling studies to determine the most effective strategies to achieve cervical cancer elimination. We examined prior commitments to disease elimination, modeling data on public health strategies for cervical cancer elimination in high and low-income countries, and possible challenges to successfully achieving elimination in the world's most vulnerable populations. Cervical cancer elimination, defined as fewer than four cases per 100,000 women, could be achievable in high-income countries (HICs) within the next 25 to 50 years. The speed and ultimate success of elimination is driven by high rates of coverage with the 9-valent vaccine, with screening providing only a small contribution. The most effective models in lowand middle-income countries (LMICs) include targeting female adolescents for two-dose vaccination, followed by either one or two lifetime screens. Challenges include a global shortage of the 9-valent vaccine, slower than anticipated roll-out to GAVI countries and low uptake rates in countries with vaccination programs. Remarkable advances in biomedical technologies have made cervical cancer elimination a potentially attainable goal within the next generation. Focusing efforts on widespread and effective implementation of evidence-based strategies to prevent the disease will be crucial. We must work to ensure that progress toward elimination is shared equally between HICs and LMICs to avoid a perpetuation of the global health disparity seen in cervical cancer.

Disclosure No significant relationships.

\section{S10.4 SELF-CARE INTERVENTIONS FOR SEXUAL AND REPRODUCTIVE HEALTH AND RIGHTS}

Manjulaa Narasimhan*. World Health Organization, Geneva, Switzerland

\subsection{6/sextrans-2019-sti.52}

Among the most promising and exciting new approaches to reach Universal Health Coverage (UHC) goals are self-care interventions for health. These interventions have the potential to increase choice, when accessible and affordable, as well as opportunities for individuals to make informed decisions regarding their health and health care. Self-care interventions 